science, pharmacy and radiation biology assembled in one book. Those involved in the development and control of sterilisation processes should find it worthwhile to have this book readily available.

J. G. SHOESMITH

\title{
Anaerobic infections
}

By A. T. Willis and K. D. Phillips. P.H.L.S. Monograph No. 3. 1983. 2nd ed. H.M. Stationery Office, London. Pp. vii and 54. £3.50.

The decade since the first edition of Dr Willis' monograph has seen an exponential growth in understanding of anaerobic infections. Dr Willis and his team have played a central role in the educational process and this completely re-written edition is particularly welcome. The increased recognition of the importance of non-sporing anaerobes is reflected in a change of emphasis in the new edition.

Much of the material in this short book with its direct, often telegraphic style is presented in tables and graphs. There are six chapters dealing with anaerobic genera of clinical importance, diseases due to anaerobes, methods of culture, diagnosis of anaerobic infections, media and methods, and gas-liquid chromatography (GLC). The summary of anaerobic infections draws attention to the wide variety of endogenous infections with non-clostridial anaerobes in the head and neck, lungs, genital tract and other soft-tissue sites as well as in the abdomen. The chapter on culture methods stresses that a well-controlled anaerobic jar system is entirely adequate for routine diagnostic microbiology and gives advice on anaerobic cabinets for laboratories with sufficiently heavy workloads to justify their use. The clinical indications of anaerobic infection, the collection and transport of specimens and the laboratory approach to the isolation and identification of anaerobes are outlined briefly with most emphasis on the early recognition of the presence of anaerobes rather than specific identification that may take several days. The chapter on media makes this an invaluable handbook for the diagnostic laboratory, although some workers may be less enthusiastic about neomycin as the preferred selective agent for non-clostridial anaerobes. The last chapter gives a realistic indication of the role of GLC in the diagnostic laboratory for direct examination of specimens and as an aid to identification of purified isolates.

Unfortunately, the chapter on classification provides a contrast between an excellent outline of the clinically significant clostridia and a rather outdated description of the Bacteroidaceae. Species of the $B$. fragilis group are still referred to as "subspecies" and all pigmented bacteroides are regarded as " $B$. melaninogenicus", which perpetuates the emphasis upon pigment production as a key characteristic. Space is wasted by devoting six pages to very simple diagrams and then repeating the information in dichotomous keys or tables. The inclusion of Campylobacter jejuni is also surprising; neither its laboratory requirements as a microaerophile nor its role in disease bear any relationship with the anaerobes. These criticisms notwithstanding, this monograph will find a welcome place amongst the "bench handbooks" in the diagnostic laboratory.

B. I. DUERDEN

\section{Fungal infection in the compromised patient}

Edited by D. W. Warnock and M. D. Richardson. 1982. John Wiley and Sons Ltd, Chichester. Pp. xi and 260. $£ 16.75$.

This is a book on systemic fungal infections, and as only species of Histoplasma, Blastomyces dermatitidis, Coccidioides immitis and Paracoccidioides brasiliensis are usually regarded as capable of infecting the normal healthy person, the vast majority of mycoses are opportunistic. Since they are not only described but lavishly illustrated in a steadily increasing number of textbooks, the worth of this book lies in accounts of defective immunity in infections with fungi, the value of serological tests in diagnosis, and the preferred treatments of the various authors.

In the chapter on aspergillosis it is stated that "Allergic complications of spore inhalation, such as asthma, extrinsic allergic alveolitis or allergic bronchopulmonary aspergillosis, rarely 
pose management problems in the immunocompromised host". The allergic complication of aspergillus spore inhalation is asthma, an accumulation of secretion in the terminal bronchioles and an exacerbation of the condition when the secretion becomes colonised by the fungus. The allergy to aspergillus ensures a compromised patient. The term allergic bronchopulmonary aspergillosis was coined by Hinson, Moon and Plummer in 1952 and it is astonishing that in what seems to be a description of this syndrome there is no mention of the skin-prick test. Without an immediate positive skin-prick test to an aqueous extract of Aspergillus fumigatus, the premier requirement for the clinical diagnosis of this condition has not been met.

The authors of the different chapters have produced a series of useful reviews and assembled a collection of references that should be of interest to clinicians, clinical immunologists, medical microbiologists interested in infection and immunity, and students preparing for the Membership Examinations of the Royal College of Pathology. It is not expensive and is to be recommended for hospital and medical school laboratories. I am pleased to have a reviewer's copy.

R. R. DAVIES

\section{Immunofluorescence techniques in diagnostic microbiology}

Edited by Joan M. B. Edwards, C. E. D. TAYLOR and A. H. Tomlinson. PHLS Monograph No. 18, 1982. H.M. Stationery Office, London. Pp. ix and 117. £3.50.

This monograph is a concise but, in parts, outdated report of immunofluorescence techniques (FAT) used in the Public Health Laboratory Service (PHLS) for the diagnosis of infections with various pathogenic bacteria, spirochaetes, protozoa, fungi, chlamydiae and viruses, by the detection of antigen or antibody. The text includes practical details of techniques appropriate for the type of organism, guidance on control tests and interpretation of results, general information on fluorescence microscopy and the technique for fluorescein-labelling of antiserum. Helpfully, methods for the preparation of antisera are given where commercial sources are not available. The appendices include a list of commercial sources of reagents and equipment, but some important sources of reagents for viral diagnosis are omitted. Some examples are Wellcome Reagents, who have made valuable contributions in the field of immunofluorescence reagents for rapid viral diagnosis, the Division of Microbiological Reagents and Quality Control of the PHLS and the European Group for Rapid Viral Diagnosis.

The pitfalls of immunofluorescence and the methods for avoiding them, e.g., non-specific fluorescence of formalin-fixed paraffin-embedded tissue sections (trypsin treatment is not mentioned), $\mathrm{Fc}$ receptors in cells infected with the herpesvirus group and the crucial importance of quality-controlled specific reagents, are mentioned but merit more emphasis and practical advice than has been given, probably in the interests of brevity.

The sections concerned with viral diagnosis by detection of antigen are brief and important viruses such as members of the herpesvirus group and measles have been omitted. Conflicting statements concerning the time of harvesting of virus to make antigen preparations for antibody testing - advanced stage of CPE in Section 7 and before the development of CPE in Section 11, the latter being more generally satisfactory-may confuse readers inexperienced with FAT. In Section 7, dealing with the detection of respiratory virus antigens in cells of nasopharyngeal secretions, the statement that the test is invalidated if fluorescence of respiratory syncytial (RS) virus antigens is not seen in ciliated epithelial cells is misleading, since intact cilia on respiratory epithelial cells are seldom seen after the very early stages of RS virus infection. Substitution of "respiratory" for "ciliated" would be more helpful.

In tests for specific IgM, the important problems of false positive reactions due to rheumatoid factor and false negatives due to high levels of specific IgG are mentioned by several contributors but methods for overcoming the problems vary from one section to another. A collation of the methods in Section 11, concerned with standardisation of procedures, would have been beneficial because a combination of methods is sometimes necessary.

The monograph is inexpensive, practical in its approach, and contains technical details useful for current application. However, readers should consult the quoted references, and also current publications on more recently developed techniques, to supplement the information in this booklet, because of the rapid progress made in recent years in diagnostic procedures. 